\title{
Teleophthalmology in COVID-19 era: an Italian ophthalmology department experience
}

\author{
Leonardo Mastropasqua ${ }^{1}$. Rossella D'Aloisio ${ }^{1}{ }^{1}$ - Lorenza Brescia ${ }^{1} \cdot$ Manuela Lanzini ${ }^{1} \cdot$ Jessica Bondi ${ }^{1}$. \\ Daniele Libertini ${ }^{1} \cdot$ Rodolfo Mastropasqua $^{2} \cdot$ Giada $^{\prime}{ }^{\prime}$ Onofrio ${ }^{1} \cdot$ Eduardo Zuppardi $^{1} \cdot$ Lisa Toto $^{1}{ }^{1} \cdot$ Luca Agnifili $^{1}{ }^{1}$
}

Received: 9 June 2020 / Revised: 6 August 2020 / Accepted: 7 August 2020 / Published online: 18 August 2020

(c) The Royal College of Ophthalmologists 2020

\section{To the Editor:}

We read with interest the recently published article by Teo et al. [1]. The authors state that the ophthalmology community as a whole is coping with significant changes for the management of outpatient and follow-up visit schedule during and after the lockdown period for COVID19 pandemic. These modifications could be an opportunity to explore novel strategies and protocols used in clinical setting in COVID-19 era.

While we agree with the authors, we would like to discuss about our novel hospital protocols at our Ophthalmology Clinic to manage safely and effectively patients with ocular diseases, avoiding their access to the hospital during the lockdown of our country where the outbreak was one of the largest in the world [2,3].

A telephonic health service with a dedicated telephone number was activated for patients to explain their ocular problems directly to specialist ophthalmologists; an institutional email was also provided to consent attached files of ocular images to better understand the diagnosis. The first step of our tele-triage was a tele-questionnaire administered to all subjects requiring the telephonic consultation, and contained questions concerning demographic and clinical data, as shown in Fig. 1.

These authors contributed equally: Leonardo Mastropasqua, Rossella D'Aloisio

Rossella D'Aloisio

ross.daloisio@gmail.com

1 Department of Medicine and Science of Ageing, Ophthalmology Clinic, University "G. d'Annunzio" Chieti-Pescara, Chieti 66100, Italy

2 Institute of Ophthalmology, University of Modena and Reggio Emilia, Modena 42121, Italy
All the data of the tele-questionnaire were recorded by qualified ophthalmologists at our ophthalmic first aid, and a specific Ophthalmic Tele-questionnaire Score (OTS) (Fig. 2) was calculated for each patient. Based on the medical history, tele-questionnaire data and, if available, photo documentation, the ophthalmologist suggested to the patient a topical therapy, or recommended a control visit at a hospital ophthalmological emergency service. When a topical therapy was suggested, patients were re-called to verify the symptoms resolution at a pre-established interval, which was based on the nature of symptoms reported. In case a concomitant systemic therapy was deemed necessary, the patient was recommended to manage any side effect with his general practitioner. When analysing the primary motivations that induced patients to require an ophthalmology tele-triage, we found that, overall, the most common ones were ocular surface-related symptoms (Table 1). In more than $95.2 \%$ of cases, the tele-intervention was useful to completely solve the clinical questions reported by patients, with symptoms disappearing with a mean time of $5.1 \pm 3.5$ days.

Extra-ocular-related motivations, such as anxiety and loneliness feelings accounted for less than $3 \%$ of the entire sample. Indeed, Coronavirus disease pandemic has become a challenge to psychological resilience and anxiety $[4,5]$.

If a bilateral conjunctival congestion was referred we accurately asked about other symptoms such as cough or fever and if so we suggested to call their general practitioner as well. After tele-survey we obtained a final score (OTS). The lower score, the better referred clinical signs. The highest OTS was found in elderly people, as expected.

In this dramatic psychological picture, the empathy of the healthcare professional supporting population through telemedicine could be the key to assure general mental health as well as to manage and treat patients in a remote location [6].

The use of telemedicine in Ophthalmology could be very useful to face epidemic conditions and also to streamline long hospital waiting lists in routine real life. 


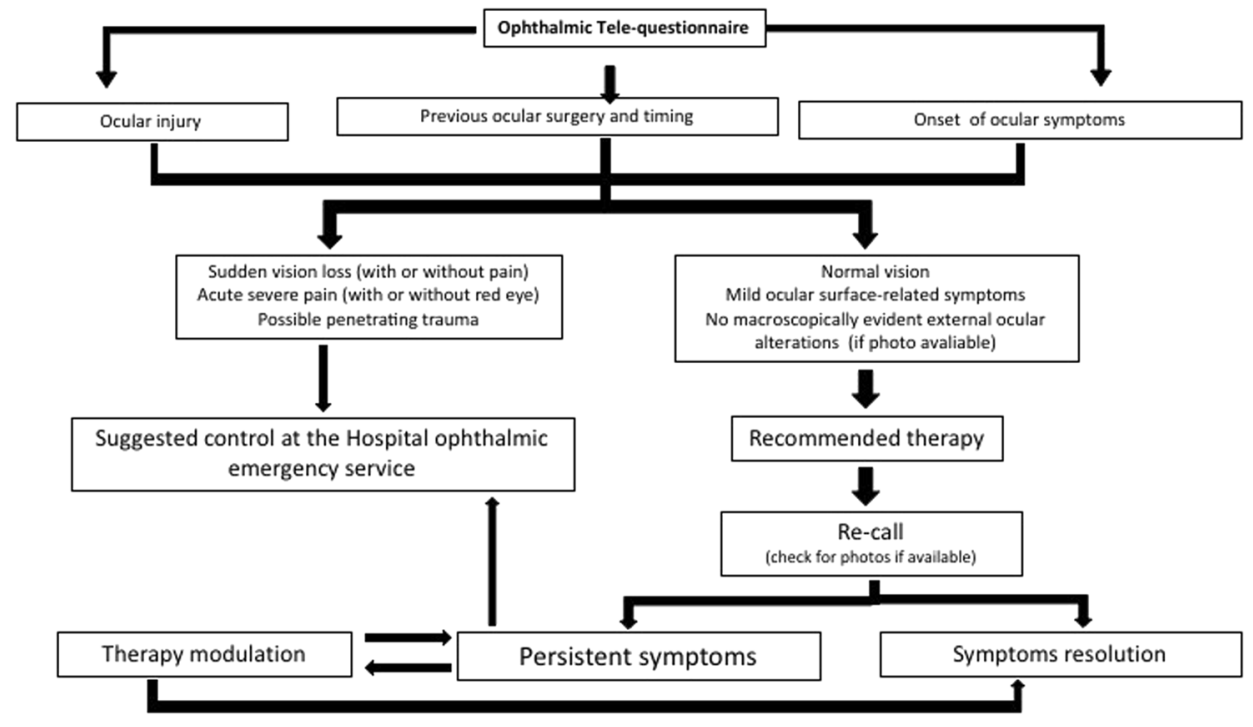

Fig. 1 Representative flowchart of emergency protocol. The first step of our tele-triage was a tele-questionnaire. The flowchart describes the questions administered to all subjects requiring the telephonic consultation concerning demographic and clinical data.

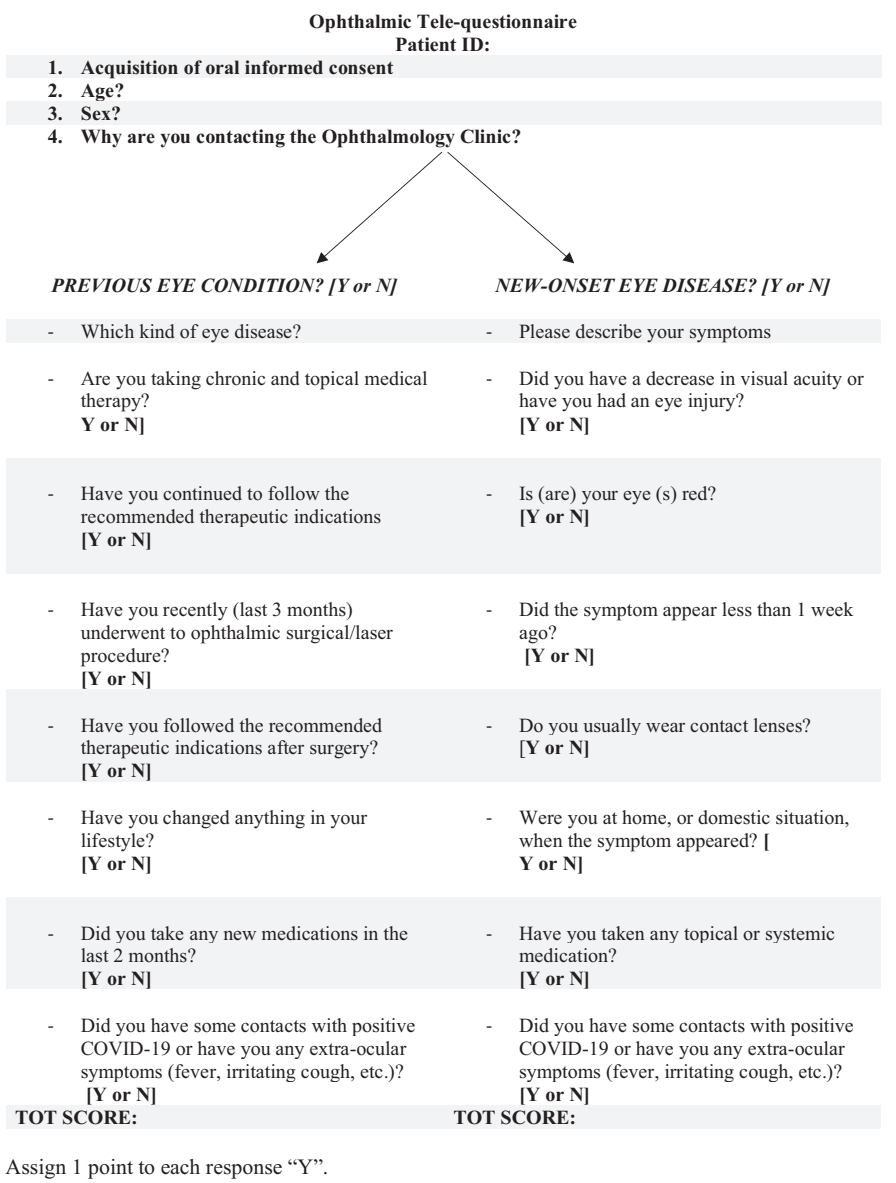

Fig. 2 Tele questionnaire to patients during tele triage. A specific Ophthalmic Tele-questionnaire Score (OTS) was calculated for each patient using a total sum, assigning one point for each "yes" response $(\mathrm{Y})$ and no point for each "no" response $(\mathrm{N})$. 
Table 1 Demographic and clinical data of enrolled patients.

\begin{tabular}{|c|c|}
\hline Age (years): mean $( \pm \mathrm{SD})$ & $55.3( \pm 17.8)$ \\
\hline $\operatorname{Sex}(\mathrm{M} / \mathrm{F}) n(\%)$ & $88 / 142(38.3 / 61.7)$ \\
\hline \multirow[t]{2}{*}{ Geographic residence $n(\%)$} & - Intra-region 134 (58.3) \\
\hline & - Extra-region 96 (41.7) \\
\hline \multirow{13}{*}{$\begin{array}{l}\text { Primary reason for ophthalmic } \\
\text { tele-triage }(\%)\end{array}$} & - Ocular pain (18.3) \\
\hline & - Burning (16.5) \\
\hline & $\begin{array}{l}\text { - Conjunctival congestion and } \\
\text { redness (15.6) }\end{array}$ \\
\hline & - Photophobia (15.2) \\
\hline & - Foreign body sensation (12.2) \\
\hline & $\begin{array}{l}\text { - Clarifications and suggestions for contact } \\
\text { lens wearers (11.9) }\end{array}$ \\
\hline & - Itchy eye (10.1) \\
\hline & - Epiphora (9.6) \\
\hline & - Eyelid cyst-like neoformation (7.8) \\
\hline & - Visual acuity reduction (7.4) \\
\hline & $\begin{array}{l}\text { - Clarifications on previous local/systemic } \\
\text { therapies }(6.5)\end{array}$ \\
\hline & $\begin{array}{l}\text { - Absence of ophthalmic symptoms, but } \\
\text { anxiety and loneliness (2.6) }\end{array}$ \\
\hline & - Other symptoms (2.2) \\
\hline \multirow[t]{14}{*}{ Ocular conditions $n(\%)$} & $143(62.3)$ \\
\hline & Previous surgery (39.2) \\
\hline & - cataract (28.2) \\
\hline & - retinal detachment (26.7) \\
\hline & - IV anti-VEGF injection (15.2) \\
\hline & - glaucoma filtering surgery (12.8) \\
\hline & - corneal transplantation (11.5) \\
\hline & - other ophthalmic surgeries (4.6) \\
\hline & Concomitant ocular diseases \\
\hline & • Glaucoma (18.5) \\
\hline & $\begin{array}{l}\text { - Corneal and ocular surface } \\
\text { diseases (16.4) }\end{array}$ \\
\hline & $\begin{array}{l}\text { - Retina diseases, not treated with IV } \\
\text { anti-VEGF injections (12.4) }\end{array}$ \\
\hline & - Diabetic retinopathy (10.3) \\
\hline & - Other (3.2) \\
\hline $\begin{array}{l}\text { Patients sent to hospital } \\
\text { ophthalmic emergency } \\
\text { service, } n(\%)\end{array}$ & $12(4.8)$ \\
\hline $\begin{array}{l}\text { Outcome for non-hospitalized } \\
\text { patients after teleintervention } \\
\text { days, } \mathrm{n}(\%)\end{array}$ & $5.1( \pm 3.5)$ \\
\hline
\end{tabular}

$I V$ intravitreal, $V E G F$ vascular endothelial growth factor.
Tele ophthalmology, in fact, is not the same as a face-toface clinical examination, however, it has become essential to cope with this nationwide public health emergency to keep safe both population and health workers. Moreover, ophthalmology field is particularly suitable for use of telemedicine in making diagnosis based on data and digital images and in providing outpatient telehealth services to people in a remote location, also in clinical practice and in primary settings.

\section{Compliance with ethical standards}

Conflict of interest The authors declare that they have no conflict of interest.

Publisher's note Springer Nature remains neutral with regard to jurisdictional claims in published maps and institutional affiliations.

\section{References}

1. Teo KYC, Chan RVP, Cheung CMG. Keeping our eyecare providers and patients safe during the COVID-19 pandemic. Eye. 2020;34:1161-2.

2. Gazzetta Ufficiale della Repubblica Italiana. 2020. https://www.ga zzettaufficiale.it/eli/id/2020/03/09/20A01558/sg.

3. Li JO, Lam DSC, Chen Y, Ting DSW. Novel Coronavirus disease 2019 (COVID-19): the importance of recognising possible early ocular manifestation and using protective eyewear. Br J Ophthalmol. 2020;104:297-8.

4. Xiao H, Zhang Y, Kong D, Li S, Yang N. Social capital and sleep quality in individuals who self-isolated for 14 days during the coronavirus disease 2019 (COVID-19) outbreak in January 2020 in China. Med Sci Monit. 2020;26:e923921.

5. Ahorsu DK, Lin CY, Imani V, Saffari M, Griffiths MD, Pakpour AH. The fear of COVID-19 scale: development and initial validation. Int J Ment Health Addict. 2020;27:1-9.

6. Tuckson RV, Edmunds M, Hodgkins ML. Telehealth. N. Engl J Med. 2017;377:1585-92. 